\title{
Analysis and experimental evaluation of the frequency response of an indoor radiating cable in the UHF band
}

\author{
Jorge A Seseña-Osorio ${ }^{1 *}$, Ignacio E Zaldívar-Huerta ${ }^{1}$, Alejandro Aragón-Zavala² and Gerardo A Castañón-Ávila ${ }^{3}$
}

\begin{abstract}
We present the modeling of the frequency response of the channel for a radiating cable system by using an autoregressive model for an indoor environment. The coefficients of the autoregressive model are determined from the experimental channel frequency response. Measurements were carried out in an indoor environment, in particular on the second floor of a university building in the frequency range of 1.3 to $1.8 \mathrm{GHz}$ by using a vector network analyzer. It is demonstrated that the use of a second-order model provides a better representation of the behavior of the channel. In this context, the coherence bandwidth and the rms delay spread show dependence with the receiver position along the radiating cable length. This dependence is crucial and must be taken into account in the design and study of broadband systems with mobility because the rms delay spread and coherence bandwidth are used to describe the time dispersion and the frequency selectivity of the multipath fading channels, respectively.
\end{abstract}

Keywords: Radiating cable; Indoor propagation; Wireless communications; Channel modeling; Autoregressive processes

\section{Introduction}

The improvement in wireless technology systems and devices has contributed to a greater concentration of mobile devices in specific locations as well as an increase in the data transmission rate. Consequently, wireless service providers and researchers are becoming more interested in obtaining the best possible performance of wireless systems under such conditions. In this context, radiating cables have been used as alternative distributed antenna systems in order to obtain optimal coverage levels in any underground or closed environment [1-4]. However, it is well-known that, for indoor wireless communications, constructive and destructive interferences have a crucial effect on the signal being transmitted. Therefore, knowledge of the behavior of the wireless channel is essential for planning and studying of any wireless systems.

A radiating cable or leaky feeder is a coaxial cable where the outer conductor has been slotted, allowing radiation to occur along the cable length for uniform coverage. Thus, radiating cables are used to distribute radio waves

\footnotetext{
* Correspondence: jaso_1@hotmail.com

${ }^{1}$ Instituto Nacional de Astrofísica Óptica y Electrónica (INAOE), Calle Luis

Enrique Erro No. 1, Tonantzintla, Puebla C.P. 72840, Mexico

Full list of author information is available at the end of the article
}

in sites where common antennas fail, besides being used as part of wireless systems such as radio detection systems and wireless indoor-positioning systems [5,6]. In recent years, the use of radiating cables has increased especially to provide cellular coverage in train and underground stations for which either macrocell penetration or an indoor distributed antenna system is not suitable. The coverage footprint provided by a radiating cable can normally be better shaped, thus filling in coverage holes in specific corridor scenarios much better than antennas and better containing leakage [3]. As prices are coming down in the manufacturing and installation of radiating cables, indoor radio solutions that include radiating cable are thus a very viable alternative nowadays. However, as stated in [3], antennas are more suitable to provide coverage in areas where the installation of radiating cables is much harder or unfeasible. On the other hand, the installation of radiating cables is a more sophisticated procedure and more costly than that of antenna installation. If coverage is to be focused on a specific area, the radiation characteristics of directional antennas can be utilized to maximize such coverage in the desired location, something that is much harder to achieve with the use of radiating cables. In summary, although radiating 
cables are a good alternative for some specific indoor environments such as corridors, there are other instances where antennas still are the preferred choice.

In order to accurately estimate the coverage and expected data rate that could be obtained using a radiating cable system, channel modeling needs to be performed. Narrowband modeling will allow engineers and designers to estimate local mean coverage if the radiating cable is installed in a venue. This issue is particularly important for voice systems, where full received signal strength and signal-to-interference and noise ratio (SINR) are important parameters that affect the performance of the system. However, if broadband data communications are to be deployed, it is very important that wideband channel characteristics be modeled, and hence, the maximum achievable data rate can be determined. Herein lies the main focus of this paper.

In [7], a typical narrowband propagation model for radiating cable systems is reported which takes into account the coupling and longitudinal losses of the radiating cable. At the same time, the slow and fast variations of the received signal levels are modeled by a log-normal distribution and a Rayleigh or Rician distribution, respectively. In the case of the signal being considered as a narrowband signal, such mentioned propagation models could be sufficient to design and study a radiating cable system. However, if a digital communication is being considered with high speed of data transmission, it is necessary to study the frequency response and the rms delay spread of the channel. In this context, little attention has been paid to such issues in radiating cable systems. For example, in [7] and [8], only the rms delay spread values have been reported and not it's modeling. The frequency response modeling allows classifying the channel as frequency selective or flat fading channel by calculating the coherence bandwidth, and the rms delay spread allows knowing the limits of the transmission rate. This fact is very important in most modern technologies because such technologies require wide bandwidths and hightransmission rate. In summary, a wideband propagation model allows knowing some system characteristics, for example, the frequency selectivity, intersymbol interference, and error floor.

The modeling of wideband channel can be developed by using time-domain or frequency-domain measurements, where the direct measurement of the impulse response or the frequency response of the channel is carried out, respectively. Time-domain measurement methods require various parameters to describe wideband channels but do not provide information of the signal phase. In contrast, using a frequency-domain measurement system, the magnitude and phase of the signal are known. Furthermore, an autoregressive model can be used to represent the channel with fewer parameters than in the case of time- domain measurements. For indoor measurements, the frequency-domain approach yields very good results, having limited applicability for outdoor measurements since the coax cable length is a strong limitation for this.

To the best of the authors' knowledge, we report for the first time the autoregressive modeling of the frequency response of channel for a radiating cable system, as well as the $r m s$ delay spread $\left(\tau_{\text {rms }}\right)$ which showed a dependency with the receiver position along the cable length. Autoregressive (AR) models in the frequency domain for indoor radio propagation have been reported in different studies $[9,10]$; however, such studies were based on wireless systems where the receiver and transmitter used conventional antennas. In contrast, the results of this work are for a wireless system which uses a radiating cable. Results showed that a second order of the autoregressive model gave the best fit to the complementary cumulative distribution of the coherence bandwidth (the 3- $\mathrm{dB}$ width of the frequency correlation function) and showed a better description of the signal delay.

The paper is organized as follows: Section 2 gives the methodology used in this study as well as the general description of the measurements and the autoregressive modeling of the channel frequency response. Section 3 is devoted to the analysis of results along with a brief discussion about the importance of the dependency of rms delay spread with the receiver position along the cable length. Finally, conclusions are given in Section 4.

\section{Methodology, description of measurements, and the frequency-domain channel modeling}

\subsection{Methodology}

In order to model the frequency response of the channel, the methodology developed in this study is depicted in Figure 1. The comparison of results is carried out by calculating the coherence bandwidth of the channel. At the same time, the rms delay spread was calculated in order to understand the relation between model parameters and the signal delay in the channel. A brief methodology description is given below.

First, the experiment was designed by selecting the components, the configuration of the devices, and the sites or regions available for the development of frequency response measurements. Secondly, the assembly of the measurement system was made as well as its respective calibration. Then, the measurements were carried out and their positions were recorded. Once the frequency responses were obtained, the coherence bandwidth and the impulse response were calculated by using the autocorrelation function and the inverse discrete Fourier transform, respectively. The rms delay spread was then calculated by using the impulse response. The model parameters were obtained from measurements, and the autoregressive model was applied in each room. Finally, simulated 


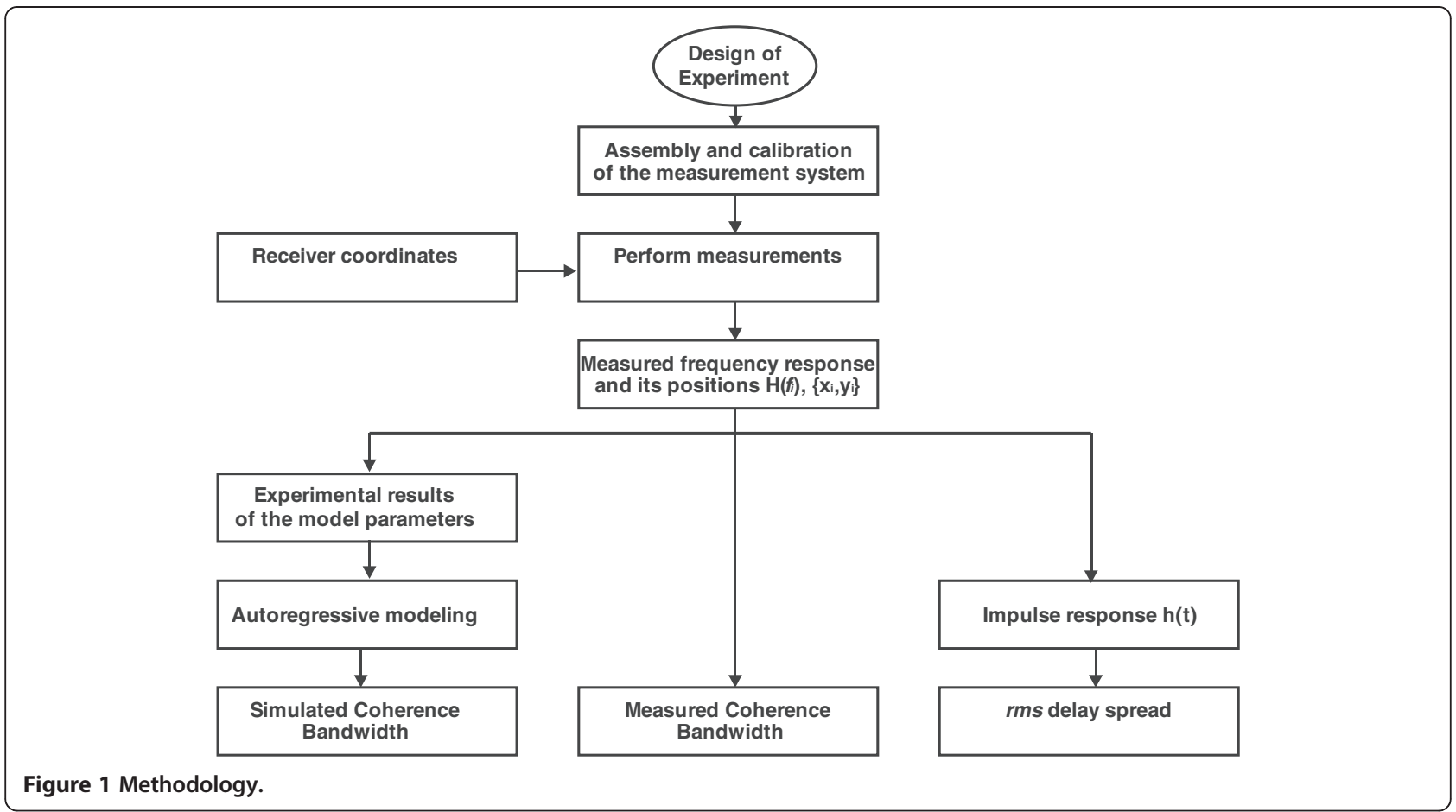

frequency responses and coherence bandwidth were calculated.

The complex frequency response of the channel is given by [11]:

$$
H(f)=\sum_{i=1}^{L} \beta_{i} \exp \left(-j 2 \pi f \tau_{i}\right) \exp \left(-j \varphi_{i}\right)
$$

where $\beta_{i}, \tau_{i}$, and $\phi_{i}$ represent the magnitude, arrival time, and phase, respectively, of the $L$ individual paths between the transmitter and receiver. Meanwhile, the channel is considered slowly time-varying. In practice, the response frequency of the channel can be measured by using a network analyzer, which sweeps the channel at discrete frequencies and measures the complex samples of the frequency response. Thus, the samples of the complex frequency response of the channel is given by:

$$
\begin{aligned}
H(k) & =\left.H\left(f_{k}\right)\right|_{f_{k}=k \Delta f}=\left.\sum_{i=1}^{L} \beta_{i} \exp \left(-j 2 \pi f_{k} \tau_{i}\right) \exp \left(-j \varphi_{i}\right)\right|_{f_{k}=k \Delta f} \\
& =\sum_{i=1}^{L} \beta_{i} \exp \left(-j 2 \pi k \Delta f \tau_{i}\right) \exp \left(-j \varphi_{i}\right), \quad 0 \leq k<N
\end{aligned}
$$

where $N$ is the number of complex samples of the frequency response and $\Delta f$ is the frequency sample spacing. If it is assumed that $f_{0}=0$, then Equation 2 is the baseband complex frequency response of the channel. Because the measurement system is band limited, the system measures windowed frequency characteristics of the channel. Therefore, the measured frequency response is given as:

$$
H_{\text {meas }}(k)=W(k) \sum_{i=1}^{L} \beta_{i} \exp \left(-j 2 \pi k \Delta f \tau_{i}\right) \exp \left(-j \varphi_{i}\right), \quad 0 \leq k<N
$$

where $W(k)$ are the effects of filtering in the frequency domain.

One method to calculate the rms delay spread $\left(\tau_{\mathrm{rms}}\right)$ is by using the impulse response of the channel which is obtained with the inverse discrete Fourier transform of Equation 3, where the time span is $T_{\mathrm{m}}=1 / \Delta f$, and the time resolution $\Delta t$ is obtained by using the inverse of the bandwidth of the measurements. Thus, the rms delay spread is the second central moment of the channel impulse response and is given by [11]:

$$
\tau_{r m s}=\sqrt{\overline{\tau^{2}}-(\bar{\tau})^{2}}
$$

where $\bar{\tau}$ is the mean excess delay and $\overline{\tau^{2}}$ is the second moment given as:

$$
\overline{\tau^{n}}=\frac{\sum_{i=1}^{L} \tau_{i}^{n}\left|\beta_{i}\right|^{2}}{\sum_{i=1}^{L}\left|\beta_{i}\right|^{2}} \quad n=1,2
$$

where $\beta_{i}$ and $\tau_{i}$ represent the amplitude and delay of the $i$ th path. 
The coherence bandwidth $\left(B_{c}\right)$ is a measure of the grade of similarity or coherence of the channel in the frequency domain. $B_{\mathrm{c}}$ can be obtained with the $3-\mathrm{dB}$ width of the magnitude of complex autocorrelation function of the frequency response which is given by [11]:

$$
R_{H}(k)=\frac{1}{N} \sum_{i=1}^{N-k} H_{\text {meas }}^{*}\left(f_{i}\right) H_{\text {meas }}\left(f_{i-k}\right), \quad k \geq 0
$$

where $H_{\text {meas }}^{*}\left(f_{i}\right)$ is the complex conjugate of the frequency response at $f_{i}$.

Frequently, there is an inverse relationship between the rms delay spread and the coherence bandwidth of the channel. The coefficients of the inverse relationship are calculated by a linear regression (on logarithmic scales) between the rms delay spread and the 3- $\mathrm{dB}$ width of the correlation function of the frequency response. This is further expanded in Section 3.

\subsection{Description of the radiating cable system}

The radiating cable used in this experiment was a RADIAFLEX ${ }^{\bullet}$ model RCF $12-50 \mathrm{~J}$, manufactured by Radio Frequency Systems RFS (Hanover, Germany) [8] and terminated with a matched $50-\Omega$ load to avoid any unwanted reflections. The radiating cable system was located inside a university building which has classrooms, laboratories, offices, and a warehouse. This building is a five-story structure where the interior and exterior walls were built
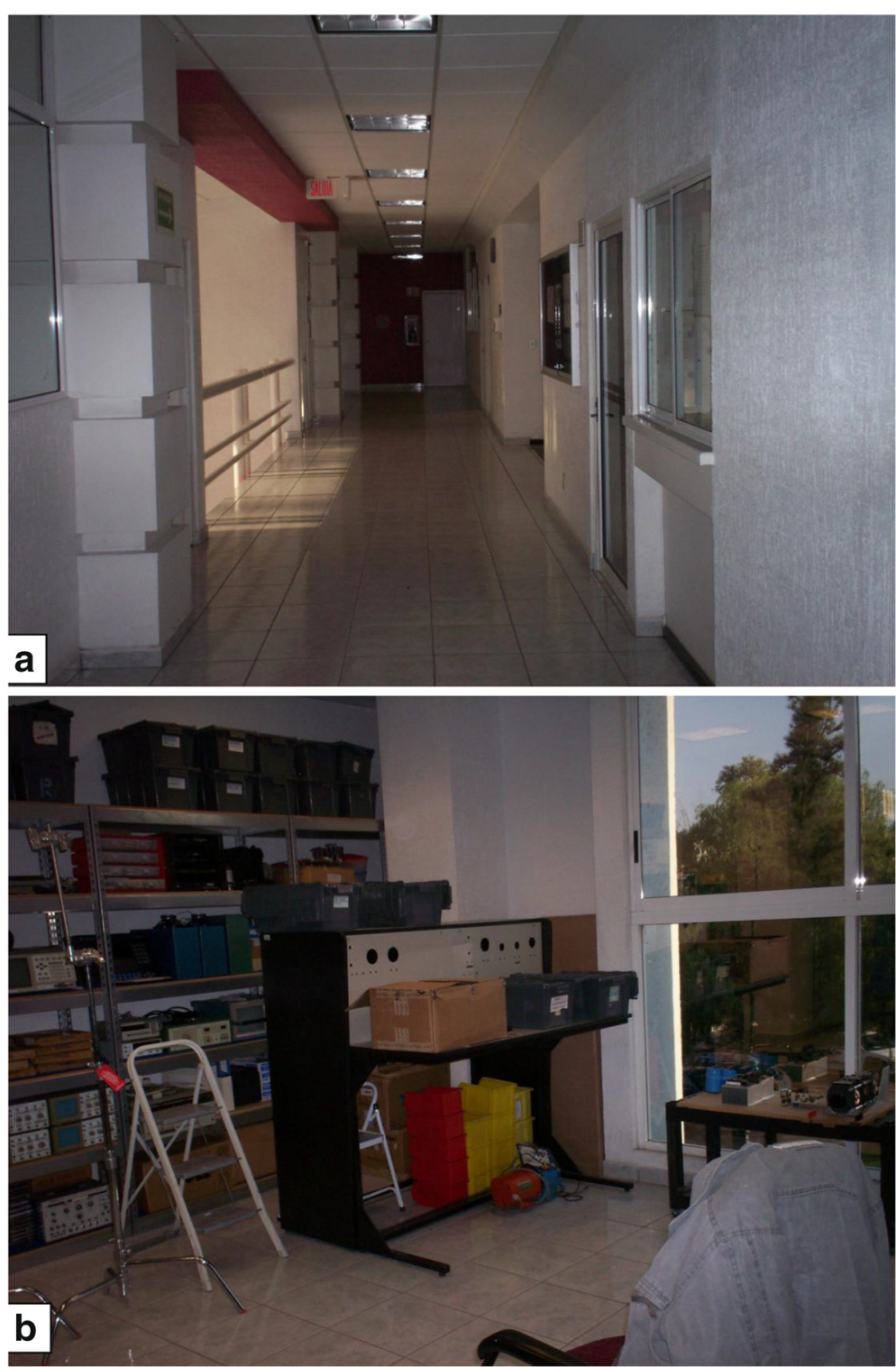

Figure 2 Corridor (a) and warehouse (b) in the Engineering and Electronic Center, Building no. 2. 
with drywall and block, respectively. Ceilings were built of steel decks and metallic beams, while the floors were built of ceramic tile. Ceilings are $4 \mathrm{~m}$ high with false ceilings of $3 \mathrm{~m}$ high. Figure 2 shows the corridor (a) and warehouse (b) of the measurement site.

The radiating cable was placed over the false ceiling of the second level, and it was laid along three paths. The first path of the radiating cable was located over the communication laboratory. The second path was positioned along the corridor, and the third path was placed over the warehouse. Figure 3 shows the layout of the second level indicating the placement of the radiating cable as well as the geographic reference used in this experiment. The communication laboratory has metallic shelves with typical equipment for radio communications. Room 2204 contains tools and equipment to fabricate printed circuit boards. Rooms 2201, 2202, and 2207 contain school furniture such as benches, chairs, desks, and worktables. Finally, in the warehouse, there are many metallic objects, electronic components, and typical equipment such as oscilloscopes, multimeters, and power supplies.

\subsection{Measurements}

The frequency response of the channel is obtained directly by the measurement system of Figure 4 . The measurement system uses as a main device a vector network analyzer (VNA). The VNA is the ZVL6 model from Rohde and Schwarz (Munich, Germany). The radiating cable is plugged to port 1 of the VNA which applies a sweep of discrete frequencies $f_{k}$; where $f_{k}=f_{0}+k \Delta f, 0<k<N$, $f_{0}$ is the lowest frequency in the band of study, $\Delta f$ is the frequency sample spacing, and $N$ is the number of frequency samples. At the same time, $N$ complex samples of the frequency response $H(k)$ are measured in the receiver antenna which is connected to port 2 of the VNA.

The measurements were achieved with $f_{0}=1,300 \mathrm{MHz}$ and $\Delta f=0.5 \mathrm{MHz}$. The frequency response consists of 1,000 complex samples $(N)$. Around $30 \mathrm{~m}$ of flexible low-loss coax cable (LMR-400 from Times Microwave Systems, Wallingford, CT, USA) were used to connect the receiver antenna with port 2 of the network analyzer. A low-noise amplifier (ZRL-2400 from Mini-Circuits, Brooklyn, NY, USA) was used between the wideband omnidirectional antenna of the receiver and the flexible low-loss coax cable. Additionally, the receiver antenna was placed $1.5 \mathrm{~m}$ high on a trolley which carried the power source for the low-noise amplifier.

The frequency response measurements were collected at fixed locations inside each room on the second floor of the building. In each room, around 120 frequency response samples were measured and recorded, and the separation between the frequency response samples was $10 \mathrm{~cm}$. These samples were distributed throughout the test area. In the corridor case, samples were collected along three parallel paths to the second segment of the radiating cable. The surrounding environment was kept stationary during the measurements by avoiding the movement of objects and the presence of people.

\subsection{Frequency-domain channel modeling}

The measured frequency response can be understood as a random process; therefore, an AR model can map the frequency response samples into a limited number of

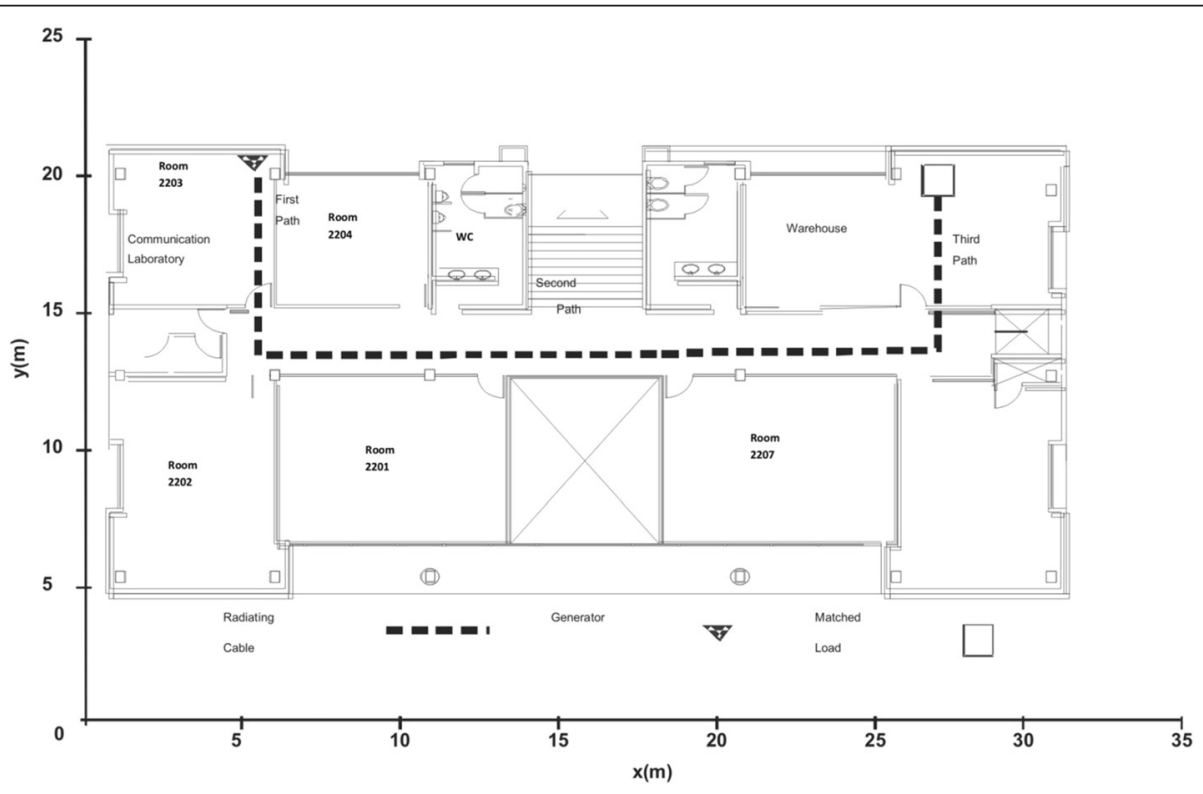

Figure 3 Layout of the second floor, showing the radiating cable position on the geographic reference. 


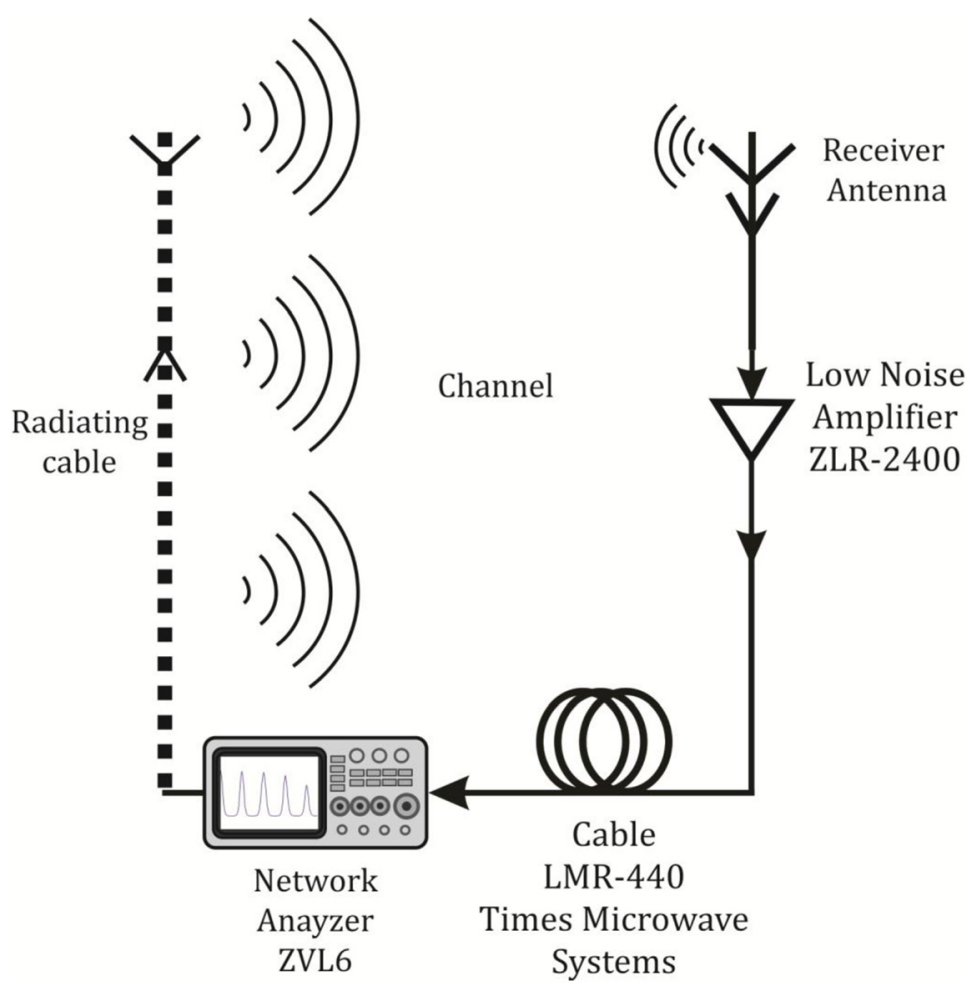

Figure 4 Wideband measurements for radiating cable system.

filter poles representing an AR process. An AR process of order $p$ is given by:

$$
H_{\text {meas }}\left(f_{n}, x\right)-\sum_{i=1}^{p} a_{i} H_{\text {meas }}\left(f_{n-i}, x\right)=V\left(f_{n}\right)
$$

where $H_{\text {meas }}\left(f_{w}\right)$ is the $n$th sample of the measured frequency response, $V\left(f_{n}\right)$ is a complex white noise process, and the complex constants $a_{i}$ are the parameters of the model. Taking the $z$-transform of Equation 7, the AR process can be depicted as the output of a linear filter with a transfer function driven by a zero-mean white noise. The transfer function is given by:

$$
G(z)=\frac{1}{1+\sum_{i=1}^{p} a_{i} z^{-1}}=\prod_{i=1}^{p} \frac{1}{1-p_{i} z^{-1}}
$$

where $p_{i}$ is the $i$ th pole of the transfer function.

The $a_{i}$ parameters are the solution for Yule-Walker equations [11]:

$$
R(-l)-\sum_{i=1}^{p} a_{i} R(i-l)=0, \quad l>0
$$

in which $R(k)$ is the frequency correlation function defined in Equation 6, and $R(-l)=R(l)$. The variance of the zero-mean white noise process $V\left(f_{n}\right)$ is the minimum mean-square of the predictor output, which is given by:

$$
\sigma_{v}^{2}=R(0)-\sum_{i=1}^{p} a_{i} R(i)
$$

In general, the order of the model depends on the measured site; however, the fifth-order process has been used as an upper bound [10].

Conventionally, a pole close to the unit circle denotes significant power at the frequency related to the angle of the pole. However, in the case of the AR frequencydomain model, a pole close to the unit circle means that there is significant power at the delay related to the pole angle. The delay is calculated as:

$$
\tau=-\frac{\theta}{2 \pi f_{s}}
$$

where $\theta$ is the angle of the pole, and $f_{s}$ is the frequency step $(0.5 \mathrm{MHz})$.

\section{Results and analysis}

Figure 5 depicts the obtained cumulative distribution of rms delay spread for all measurements. It is observed that the maximum value of the rms delay spread is $54.4 \mathrm{~ns}$ and the minimum value is $10.25 \mathrm{~ns}$. As can be observed in the results, the cumulative distribution 


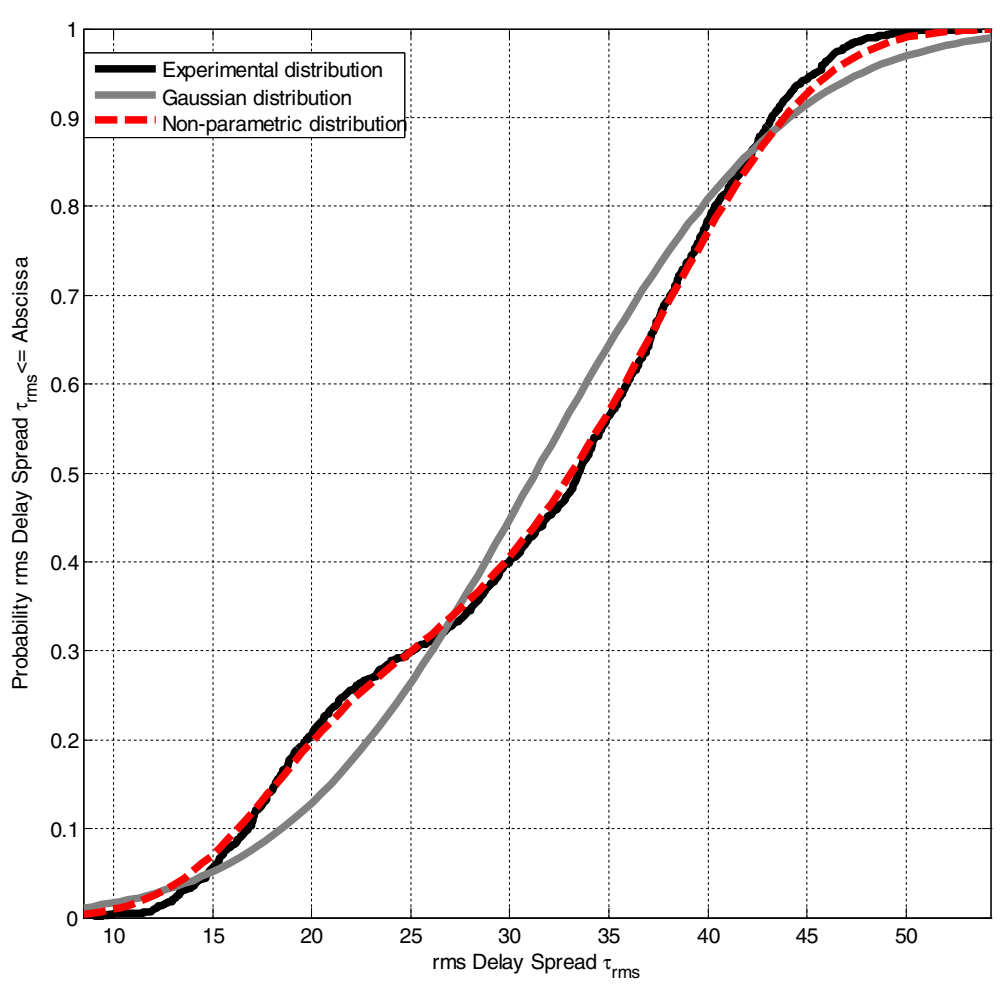

Figure $5 \mathrm{CDF}$ of the rms delay spread obtained from wideband measurements and comparison with Gaussian and non-parametric distributions.

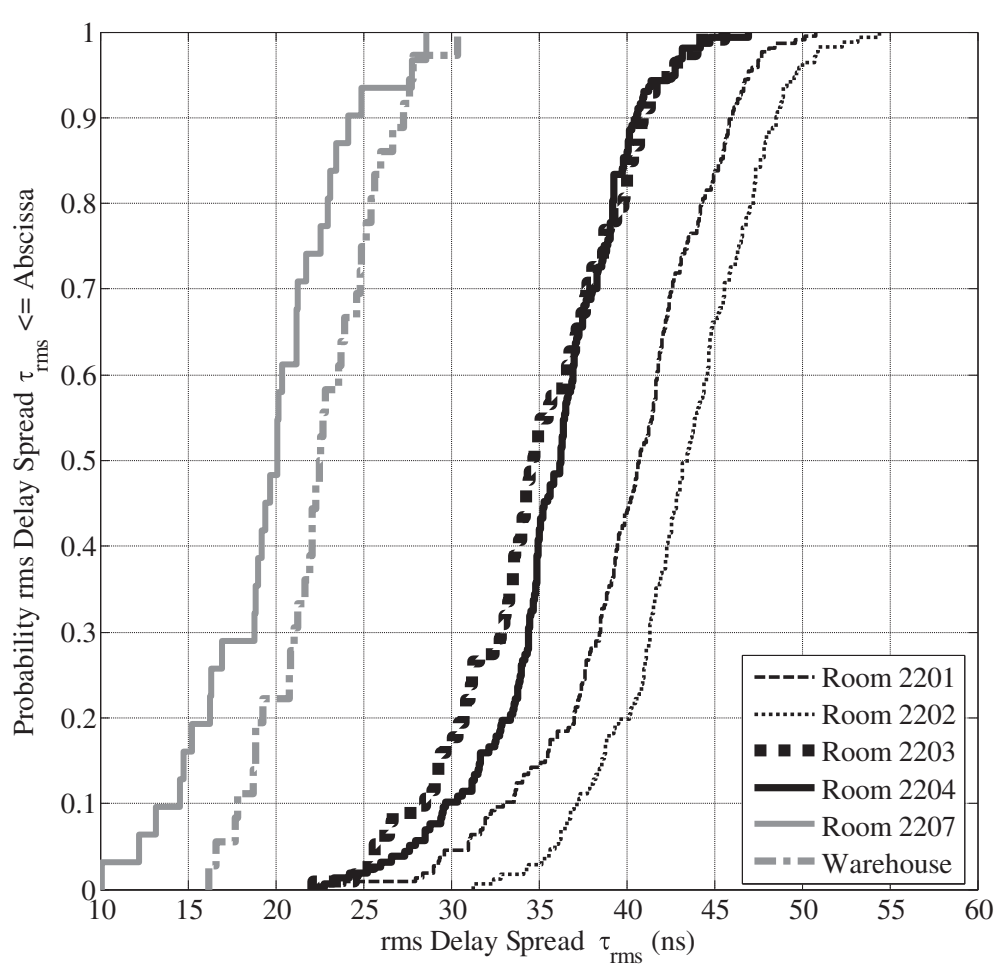

Figure 6 CDF of the rms delay spread for every room of the second floor. 

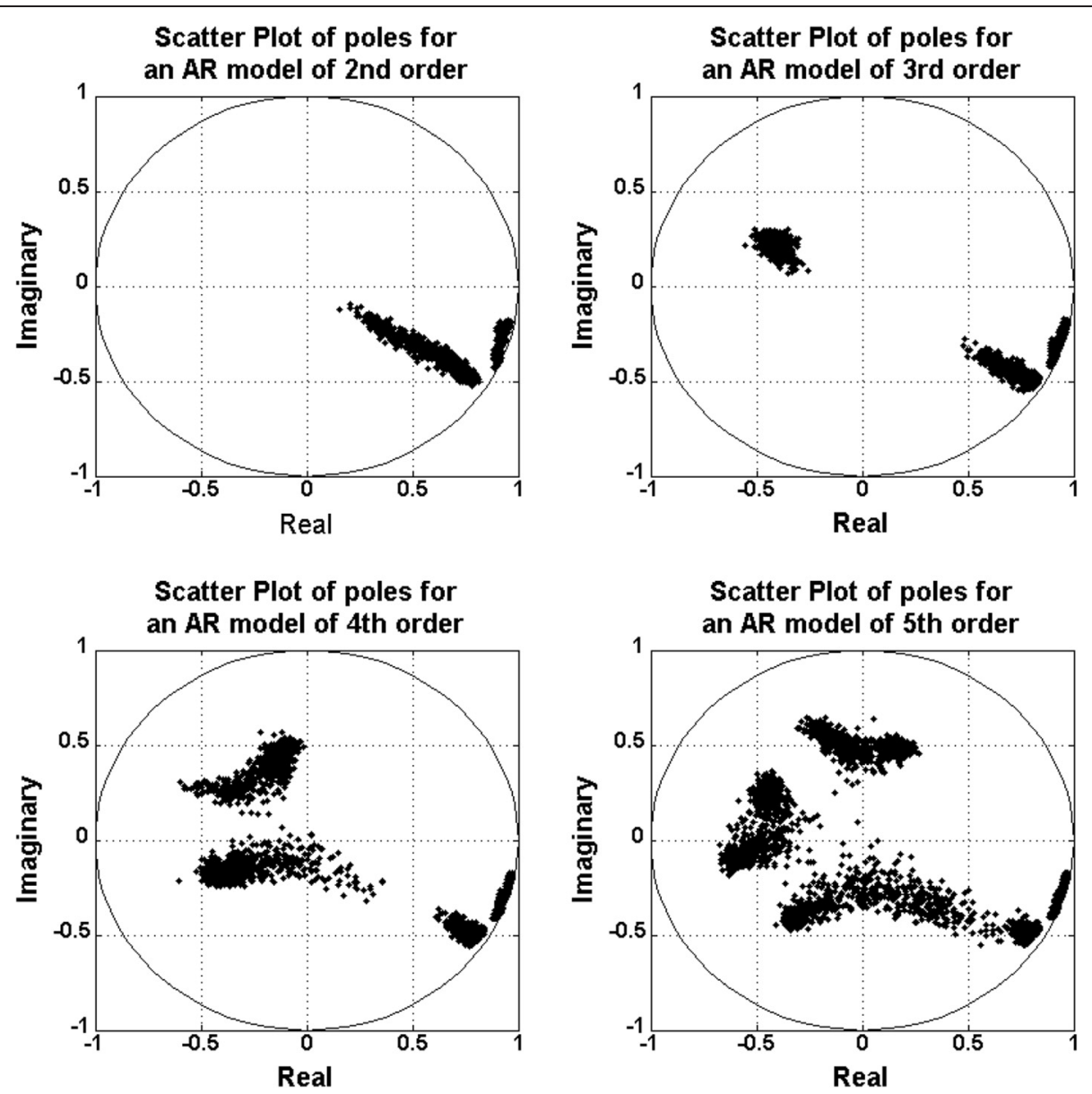

Figure 7 Scatter diagrams of the locations of the poles for the AR models of orders second, third, fourth, and fifth.

does not follow any particular distribution, and the range of rms delay spread values is closely similar to [7]. On the other hand, the cumulative distribution functions of the delay spread for every room have a smaller range of variations and different averaged values as shown in Figure 6.

After various series of measurements, the corridor was found as the main site where the general characteristics of the channel can be determined (coherence bandwidth, delay spread, impulse response variation, etc.), since the poles in the corridor are a good approximation (in almost all magnitude and phase range) to the poles obtained in all rooms. Therefore, the results depicted in Figure 7 correspond to the scatter plots of the poles for models of orders 2, 3, 4, and 5 at the corridor. These results show that for all cases, there are two poles close to the unit circle with averaged angles of -0.3403 and $-0.5394 \mathrm{rad}$ which correspond to delays of 108.32 and 171.7 ns, respectively. According to the work reported in [10], two significant poles can be interpreted as two significant clusters of multipath arrivals. In this particular case, the poles of the AR model of second order showed a betterdefined behavior in the complex plane. This betterdefined behavior showed the variation of the signal delay along the cable length. The magnitude of pole 1 was almost constant and its angle rotates clockwise, which represents the variation of the delay in receiver positions along the cable length. At the same time, pole 2 displayed a reduction of its magnitude and minimum variations of its angle. This describes the reduction of the $\tau_{\text {rms }}$ as the receiver moved away from the cable feeder in a direction parallel to the cable. Recall that in [10], the delay spread is mainly related with the angles of both poles and less closely with the magnitude of the second pole.

Figure 8 shows the complementary cumulative distribution functions of the $3-\mathrm{dB}$ width of the correlation function for the measurements of corridor and room data generated with AR models of second and fifth orders. The statistics of the AR model of second order fits more closely to the experimental measurements and will be used to understand the relationship between the model poles and the behavior of the channel. 


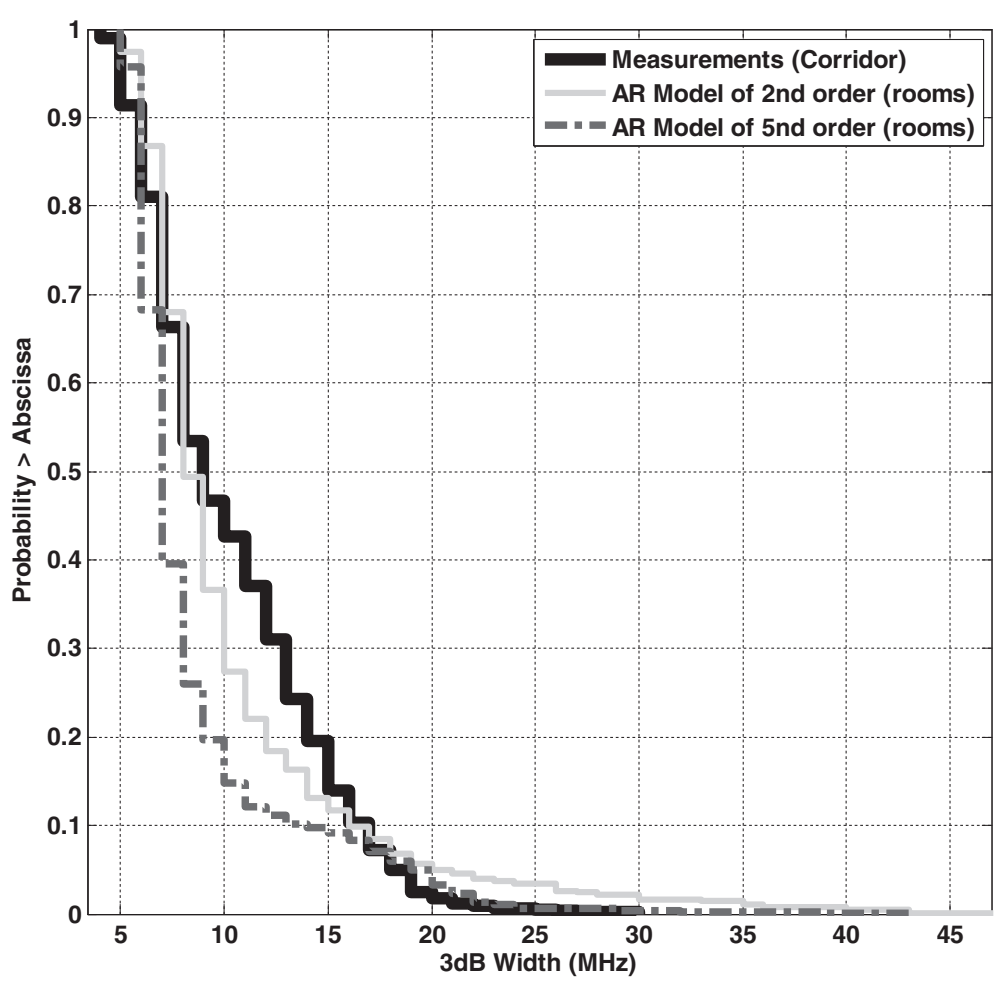

Figure 8 Complementary cumulative distribution functions of the frequency correlation function for measurements and the AR models.

Figure 9 depicts the enlarged scatter plot of poles for the second-order model. Note that the poles show a shifting which corresponds to the position change of the receiver along the corridor. The arrow marks the direction of the shifting when the receiver is moved along the positive $x$-axis from $x=5 \mathrm{~m}$ to $x=25 \mathrm{~m}$ at $y=14 \mathrm{~m}$ (according to the layout of Figure 3 ). The clockwise shifting of pole 1 marks an increase of the delay of the first cluster of multipath arrivals. Pole 2 has an almost null change in the angle, but a reduction of the magnitude is manifested. It implies a reduction of the span of the impulse response or the power delay profile along the corridor, and in consequence, the rms delay spread is reduced. This situation can be appreciated in Figure 10 from a point of view of the impulse response.

Figure 10 corresponds to the normalized impulse responses which were obtained along a parallel route to the radiating cable in the corridor. It illustrates how the impulse response starts with different delays. In the case of $x=5 \mathrm{~m}$ and $y=14 \mathrm{~m}$, the impulse response starts around $15.5 \mathrm{~ns}$; on the contrary, for $x=25 \mathrm{~m}$ and $y=14$ $\mathrm{m}$, the impulse response starts around $108 \mathrm{~ns}$ (refer to Figure 3 for both cases). Furthermore, the impulse responses finish in a similar time. This fact explains the different mean values obtained from the rms delay spread for every room (Figure 6). The former corresponds to the area of the radiating cable which is near to the feeder and the latter corresponds to the opposite situation.

Note the strong dependence between the rms delay spread and the length of the radiating cable as well as its best fit (Figure 11). This relationship along the corridor can be approximated as:

$$
\tau_{r m s} \approx 50-\frac{3}{2} x
$$

where $x$ is the position of the receiver along the corridor given in meters, and $\tau_{\mathrm{rms}}$ is given in ns. An example of this behavior can be seen in Figure 9, where the magnitude of pole 1 increases as the delay spread decreases.

It was shown in [12] that without diversity or equalization, the ratio of the rms delay spread to symbol duration in a digital transmission must be kept below 0.2 to have a tolerable intersymbol interference. Thus, assuming this criterion, in Room 2202, the maximum value of $\tau_{\text {rms }}$ was $54.4 \mathrm{~ns}$, and therefore, the channel can support a data rate up to $3.6 \mathrm{Mb} / \mathrm{s}$. Meanwhile in Room 2207 , the maximum value of $\tau_{\text {rms }}$ was $28.5 \mathrm{~ns}$; hence, the channel can handle a data rate up to $7 \mathrm{Mb} / \mathrm{s}$. Such a situation must be considered in the design of modern wireless systems.

Finally, the relationship between the coherence bandwidth $B_{\mathrm{C}}$ and rms delay spread $\tau_{\text {rms }}$ that was mentioned in 


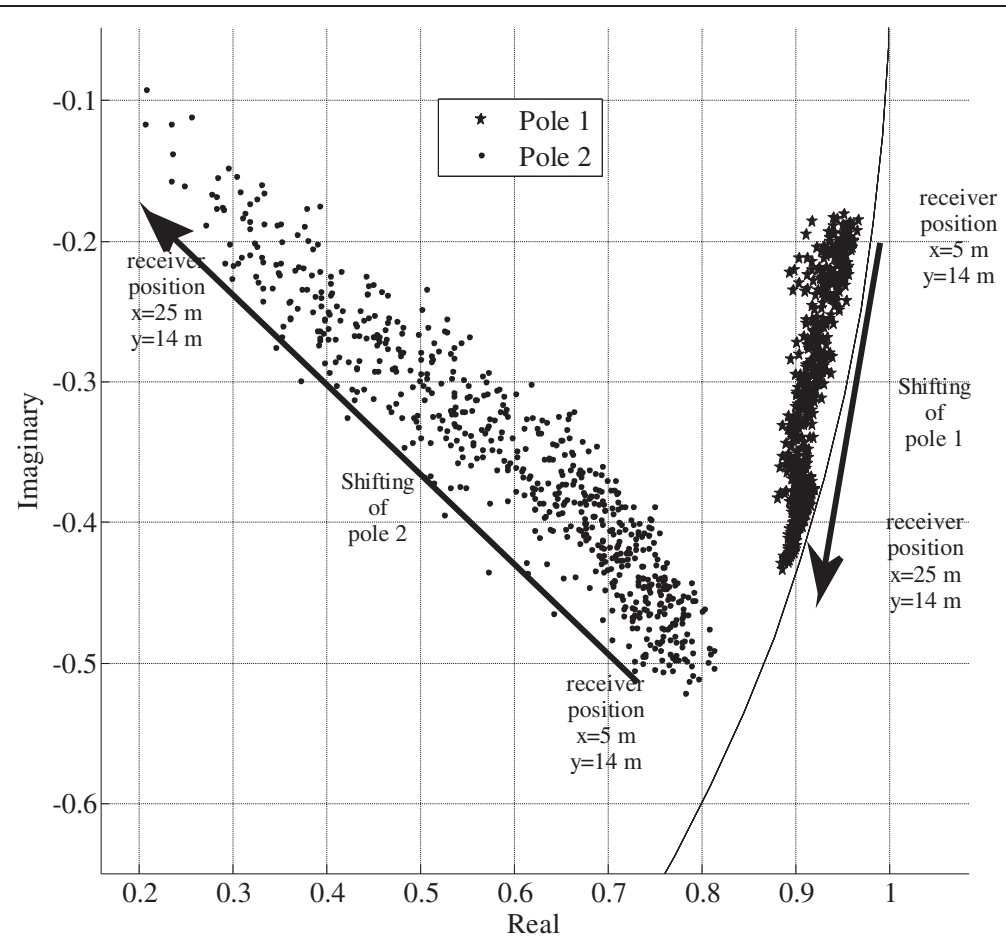

Figure 9 Enlarged scatter plot of poles for the second-order model.

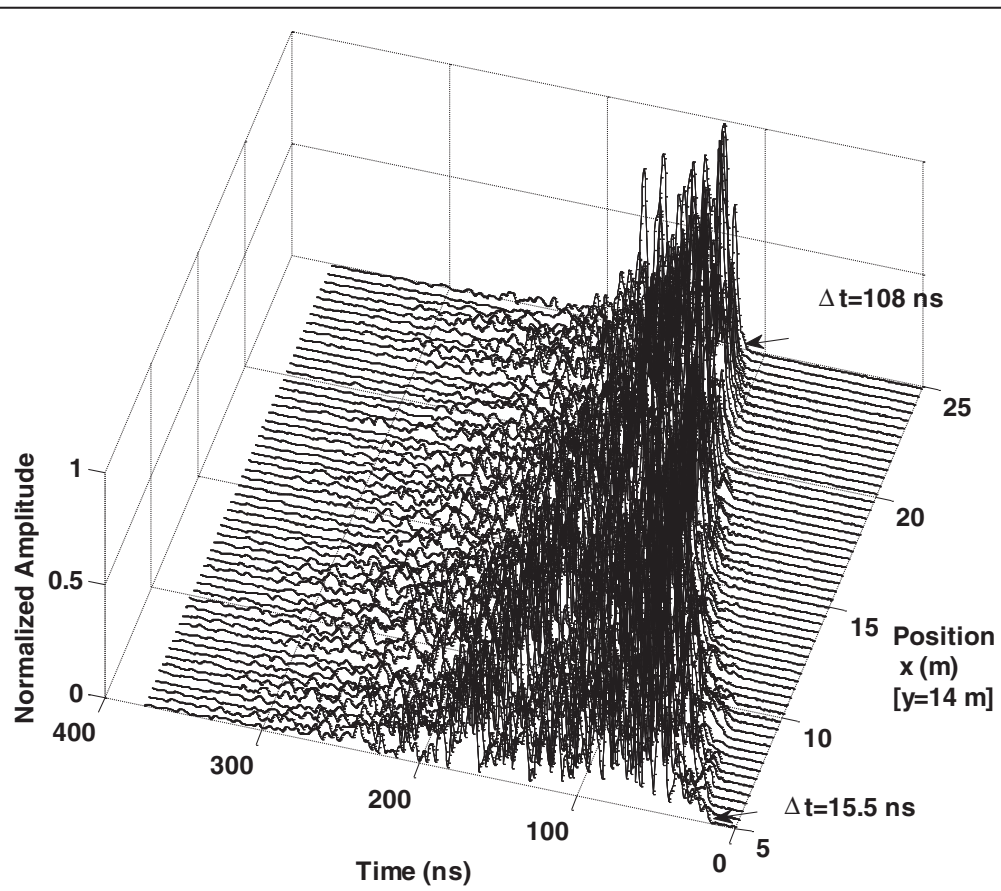

Figure 10 Normalized impulse responses along the corridor. 


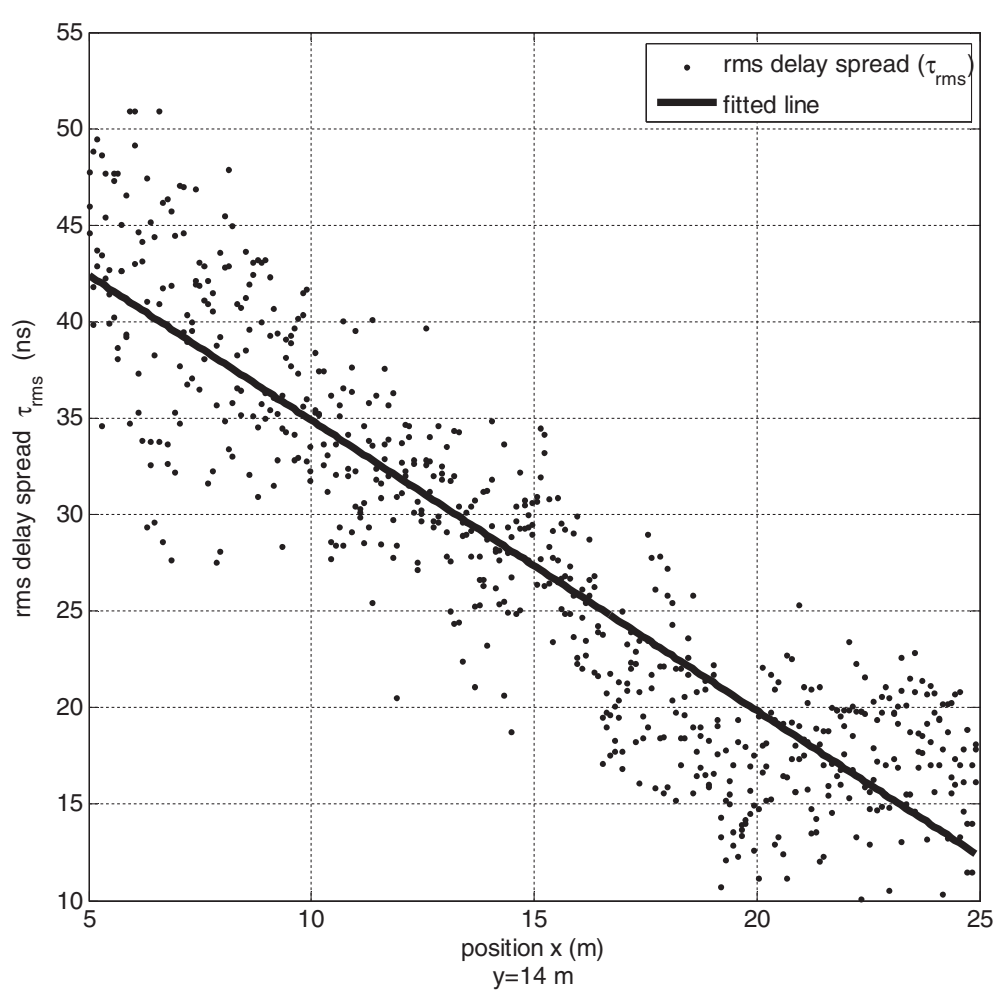

Figure 11 Best fit of scatter plot of $r m s$ delay spread $\tau_{\text {rms }}$ versus $x$ position along the corridor.

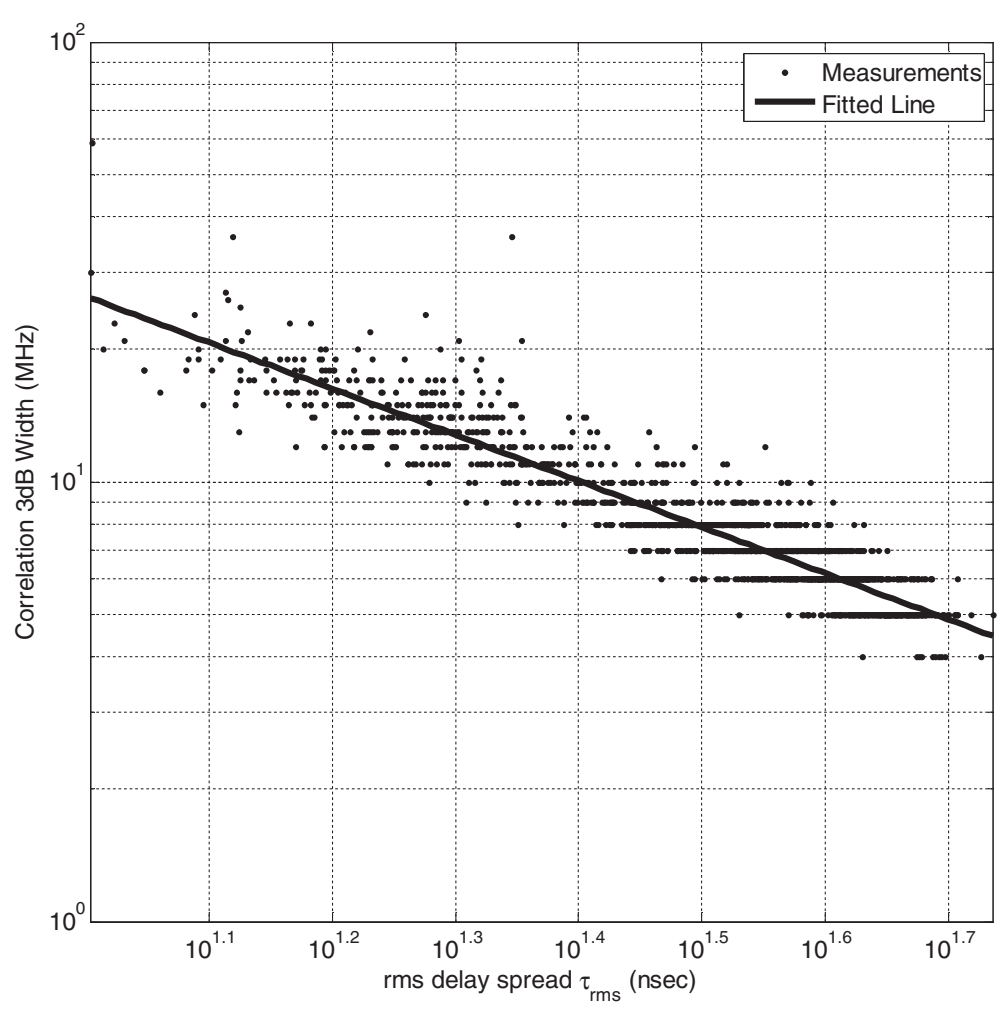

Figure 12 Relationship between $B_{\mathrm{c}}$ and $\tau_{\mathrm{rms}}$. 
Section 2 was found using a linear regression in logarithmic scale and is given by:

$$
B_{c} \approx \frac{1}{\tau_{r m s}}
$$

Figure 12 shows the width $(3 \mathrm{~dB})$ of the frequency correlation function versus its corresponding rms delay spread and the best-fit line.

\section{Conclusions}

At the beginning of this paper, it was pointed out that radiating cables can be used as part of wireless systems such as in distribution systems, by providing coverage for in-building cellular scenarios, as well as in radio detection and wireless indoor-positioning systems. Nevertheless, modern digital communication systems require broadband to be deployed. In this context, the study and design of any wireless system needs to know the multipath fading behavior of the channel in order to obtain an optimal performance of the system.

The coherence bandwidth and the rms delay spread $\left(\tau_{\text {rms }}\right)$ were obtained by measuring the frequency response of the channel, and it was demonstrated that there is dependence between $\tau_{\text {rms }}$ and the receiver position along the cable length. This dependence must be taken into account in the design of broadband systems with mobility. Furthermore, such dependence can be used for wireless localization applications in indoor environments.

Moreover, an AR model for the frequency response was carried out. Complementary cumulative distribution functions showed that a second-order AR model gives the best fit at the $3-\mathrm{dB}$ width of the frequency correlation function and showed a better-defined behavior in the complex plane. This better-defined behavior can be useful in the computer simulation of the channel or in the designing of simulation tools which allow evaluating specific systems under different schemas of modulation, coding, and equalization.

As it is known, the most modern wireless technologies are based on models because of the random variations of the wireless channel. Thus, the design and study of radiating cable systems can be carried out by using autoregressive modeling of frequency response; however, it is necessary to implement more studies in different environments in order to compare and verify the channel behavior obtained in this study, including the effects of moving scatters.

\section{Competing interests}

The authors declare that they have no competing interests.

\section{Acknowledgements}

This work was partially supported by the Mexican Consejo Nacional de Ciencia y Tecnología (CONACYT), Project No. 154691. One of the authors, Jorge A. Seseña-Osorio wishes to thank the CONACYT for Scholarship Number 34612.

\section{Author details}

${ }^{1}$ Instituto Nacional de Astrofísica Óptica y Electrónica (INAOE), Calle Luis Enrique Erro No. 1, Tonantzintla, Puebla C.P. 72840, Mexico. ${ }^{2}$ Tecnológico de Monterrey, Campus Querétaro, Epigmenio González 500, Fracc. San Pablo, Santiago de Querétaro, Querétaro C. P. 76130, Mexico. ${ }^{3}$ Tecnológico de Monterrey, Campus Monterrey, Av. Eugenio Garza Sada 2501 Sur, Col. Tecnológico, Monterrey, NL C.P. 64849, Mexico.

Received: 21 May 2014 Accepted: 6 January 2015

Published online: 10 February 2015

\section{References}

1. SEM Dudley, TJ Quinlan, SD Walker, Ultrabroadband wireless-optical transmission links using axial slot leaky feeders, and optical fiber for underground transport topologies. IEEE Trans. Veh. Technol. 57(6), 3471-3476 (2008)

2. L Feng, X Yang, Z Wang, Y Li, The application of leaky coaxial cable in road vehicle communication system, in Proceedings of 9th International Symposium on Antennas Propagation and EM Theory, 2010, pp. 1015-1018

3. I Stamopoulos, A Aragón-Zavala, SR Saunders, Performance comparison of distributed antenna and radiating cable systems for cellular indoor environments in the DCS band, in Proceedings of Twelfth International Conference on Antennas and Propagation, 2003, pp. 771-774

4. EUPEN AG Cable, URL: http://www.eupen.com/cable/rf/radiating/index.html. Accessed 12 May 2014

5. T Higashino, K Tsukamoto, D Komaki, Radio on leaky coaxial cable (RoLCX) system and its applications, in Proceedings of PIERS, 2009, pp. 40-41

6. M Weber, U Birkel, R Collmann, J Engelbrecht, Comparison of various methods for indoor RF fingerprinting using leaky feeder cable, in Proceedings of 7th Workshop on Positioning Navigation and Communication (WPNC), 2010, pp. 291-298

7. YP Zhang, Indoor radiated-mode leaky feeder propagation at $2.0 \mathrm{GHz}$. IEEE Trans. Veh. Technol. 50(2), 536-545 (2001)

8. Chehri, A., H. Mouftah, Radio channel characterization through leaky feeder for different frequency bands, in Proceedings of IEEE $21^{\text {st }}$ International Symposium on Personal Indoor and Mobile Radio Communications, Istanbul, Turkey, 26-30 Sept 2010. pp. 347-351.

9. G Gu, X Gao, J He, M Naraghi-Pour, Parametric modeling of wideband and ultrawideband channels in frequency domain. IEEE Trans. Veh. Technol. 56(4), 1600-1612 (2007)

10. SJ Howard, K Pahlavan, Autoregressive modeling of wide-band indoor radio propagation. IEEE Trans. Commun. 40(9), 1540-1552 (1992)

11. K Pahlavan, AH Levesque, Wireless Information Networks, 2nd edn. (Wiley \& Sons, Chichester, West Sussex, UK, 2005)

12. JCl Chuang, The effects of time delay spread on portable radio communications channels with digital modulation. IEEE J. Sel. Areas Commun. 5(5), 879-899 (1987)

\section{Submit your manuscript to a SpringerOpen ${ }^{\odot}$ journal and benefit from:}

- Convenient online submission

- Rigorous peer review

- Immediate publication on acceptance

- Open access: articles freely available online

- High visibility within the field

- Retaining the copyright to your article

Submit your next manuscript at $>$ springeropen.com 